Niğde Ömer Halisdemir Üniversitesi Mühendislik Bilimleri Dergisi
Niğde Ömer Halisdemir University Journal of Engineering Sciences
ISSN: 2564-6605
Araştırma / Research

\title{
Yer altına gömülü üç boyutlu cisimlerin bozulmuş born iteratif yöntemi ile tespit edilmesi
}

\author{
Reconstruction of three dimensional objects buried underground by distorted \\ born iterative method
}

\author{
Rıza Erhan Akdoğan,* (D), Kamil Fatih Dilaver' ${ }^{(i)}$, Tülün Durukan1(i), \\ Yasemin Altuncu ${ }^{1}$ (iD
}

${ }^{I}$ Niğde Ömer Halisdemir Üniversitesi, Elektrik Elektronik Mühendisliği, 51240, Niğde, Türkiye

\section{Özet}

Bu çalışmada, yer altında gömülü üç boyutlu (3B) cisimlerin geometrik ve elektriksel özelliklerinin yer üstünden yapılan ölçümlerle belirlenmesine yönelik bir elektromanyetik ters saçılma problemi ele alınmıştır. Problemin çözümü bozulmuş born iteratif yöntemi (Distorted Born Iterative Method - DBIM) kullanılarak yapılmıștır. Hava ve toprak ortamlarını birbirinden ayıran ara yüzey yukarıdan bir elektromanyetik dalga kaynă̆ ile aydınlatılmıs ve yine yukarldaki bir bölge boyunca elektrik alan ölçümleri yapılmıştır. Elektromanyetik dalga kaynağ olarak peș peșe farklı geliș açılarılla gönderilen düzlem dalgalar kullanılırken ölçümler bir düzlem boyunca birden fazla noktada alınmıştır. Illgilenilen hacimsel bir bölgede bulunan cisimlerin geometrik ve elektriksel özellikleri, bu bölgenin kompleks dielektrik sabitinin belirlenmesi sonucu ortaya çıkartılmaktadır. Sunulan yöntemin, yer altındaki rastgele geometrili üç boyutlu nesnelerin tespitindeki bașarısını göstermek için çeşitli saylsal sonuçlar sunulmuştur. Elde edilen sonuçlar, bozulmuş born iteratif yönteminin düşük ve orta düzeyde kontrasta sahip cisimlerin hem geometrik hem de dielektrik özelliklerinin belirlenmesinde oldukça başarll olduğunu ortaya koymaktadır. Yüksek kontrastl cisimlerin ise yeri ve geometrik özellikleri bașartyla bulunurken bağll dielektrik sabiti ve iletkenlikleri gerçek değerlerinden daha düşük seviyelerde bulunmuştur.

Anahtar kelimeler: Ters saçılma, Gömülü cisim, Bozulmuş born iteratif yöntemi

\section{Giriş}

Toprak altına gömülü cisimlerin geometrik ve elektriksel özelliklerinin belirlenmesine yönelik ters elektromanyetik saçılma problemleri, yer altındaki madenlerin, fay hatlarının, arkeolojik kalıntıların, tünellerin ve mayınların tespit edilmesi gibi çok geniş uygulama alanlarına sahiptir. Yanına yaklaşılamayan bir cismin, geometrik ve elektriksel özelliklerinin uzaktan yapılan ölçüm verileri kullanılarak belirlenmesi yeniden yapılandırma (rekonstrüksiyon) olarak ifade edilir.

Bir ters saçılma uygulamasında ele alınan problem geometrisinin ve elektriksel özelliklerinin belirli bir eksen boyunca değişmediği durumlarda problem iki boyutlu skaler probleme indirgenebilmekte ve çözüm hem matematiksel hem de hesaplama açısından nispeten basitleşebilmektedir $[1,2]$. İki boyutlu yaklaşımların geçerli olduğu pek çok başarılı çalışma olmakla birlikte gerçek hayat problemlerini

\begin{abstract}
In this study, an electromagnetic inverse scattering problem to reveal the geometric and dielectric properties of three-dimensional (3D) objects buried underground by measuring from above is discussed. The solution of the problem is achieved by using the distorted born iterative method (DBIM). The interface separating the air and soil media is illuminated from above with an electromagnetic wave source and electric field measurements are conducted across a region above. While plane waves are used as an electromagnetic wave source, which are sent consecutively with different arrival angles, measurements are taken at more than one point along a plane. The geometric and electrical properties of objects in a volumetric region of interest are revealed by determining the complex dielectric constant of this region. Various numerical results are presented to show the success of the presented method in detecting threedimensional objects with arbitrary geometry buried into earth. The results show that the distorted born iterative method is very successful in determining both geometric and dielectric properties of objects with low and medium contrast. While the location and geometric properties of high contrast objects are successfully found, the relative dielectric constants and conductivities are found to be lower than their real values.
\end{abstract}

Keywords: Inverse scattering, Buried object, Distorted Born Iterative Method

daha iyi modellemek adına problemleri üç boyutlu (3B) olarak ele almak daha gerçekçi bir yaklaşım olacaktır $[3,4]$. Tespit edilmeye çalışılan cismin özelliklerinin yanında cismin içinde bulunduğu ortamın özellikleri de ters problemlerin zorluk derecesini belirleyen önemli kriterlerden biridir. Örneğin sınırsız, homojen ortamdaki cisim ile tabakalı ortamdaki cismin tespit edilmesi arasında büyük bir fark söz konusudur. Birincisinde özellikleri belirlenmeye çalışılan cismin etrafında dolanarak her yönden aydınlatılabilme ve elektrik alanı her yönden ölçülebilme imkânı varken [5-7] ikincisinde hem uyarma hem de ölçme yalnızca üst tabakadan yapılabilmektedir. Böyle bir durumda, ölçüm verilerindeki yetersizlik veya küçük hatalar ters problemin çözümünde belirsizlik ve büyük hatalara yol açabilmekte ve tek çözüm bulmayı zorlaştırmaktadır [8-10]. $\mathrm{Bu}$ tür problemler kötü kurulmuş (ill-posed) problemler olarak adlandırılır ve ters saçılma problemleri çoğunlukla bu sinifa girerler [11].

\footnotetext{
* Sorumlu yazar / Corresponding author: Riza Erhan Akdoğan, e-posta / e-mail: ariza @ ohu.edu.tr (R.E. Akdoğan) Geliș / Recieved: 20.11.2020 Kabul / Accepted: 23.12.2020 Yayımlanma / Published: 15.01.2021

doi: $10.28948 /$ ngmuh. 828944
} 
Literatürde ters saçılma problemlerinin çözümü için geliştirilmiş çok sayıda başarılı yöntem bulunmaktadır. Bunlar arasında Born yaklaşımı (Born Approximation-BA) ve Rythov yaklaşımı (Rythov Approximation-RA) gibi doğrusal yöntemler çoklu saçılma etkilerini göz ard1 ettiğinden yalnızca zayıf saçıcı cisimlere ilişkin ters saçılma problemlerinin çözümünde etkindirler [12]. Oysaki ters saçılma problemleri yapısı gereği çoğunlukla doğrusal değildir (nonlineerdir). Bu özellikleri ters saçılma problemlerinde güvenilir, kararlı ve hesaplama açısından etkin çözümler bulmayı zorlaştırmaktadır. Bir cismin saçıcılık derecesi, cisim ile içinde bulunduğu ortam arasındaki dielektik özellikler bakımından var olan farka yani kontrasta bağlıdır. Dolayısıyla yüksek kontrastlı bir cisim yüksek saçıcılık özelliğine sahiptir ve böyle cisimlerin doğru ve hassas bir şekilde yeniden yapılandırılması için doğrusal olmayan yöntemler kullanılmak zorundadır. Genel olarak, doğrusal olmayan yöntemler, yinelemeli (iteratif) yöntemler olup uygun şekilde tanımlanan bir hata fonksiyonunun iteratif minimizasyonuna dayanırlar. [13].

Born iteratif yöntemi (Born Itertive Method-BIM) ve bozulmuş BIM (Distorted Born Itertive Method DBIM) yaygın olarak kullanılan doğrusal olmayan ters saçılma yöntemleridir [14-16]. BIM'de, integral denklem içinde yer alan Green fonksiyonu her iterasyon adımında sabit tutulurken arka plan alanı için born yaklaşımı kullanılır. Buna karşın DBIM'de saçıcı cisim homojen olmayan zemin uzayında bir bozucu olarak kabul edilerek bağıl dielektrik sabitindeki fark ve Green fonksiyonu her iterasyon adımında güncellenir [17]. Bunlara ilaveten kontrast kaynak inversiyon (Contrast Surce Inversion - CSI) [18] ve alt uzay optimizasyon yöntemi (Subspace Optimization Method SOM)'de [19] sıklıkla kullanılan ve iyi sonuç veren yöntemlerdendir.

$\mathrm{Bu}$ çalışmada, yer altında gömülü $3 \mathrm{~B}$ cisimlerin geometrik ve elektriksel özelliklerinin yer üstünden yapılan ölçümlerle belirlenmesine yönelik bir elektromanyetik ters saçılma problemi çözülmüştür. Problemin çözümü doğrusal olmayan bir ters saçılma algoritması olan DBIM algoritması kullanılarak yapılmıştır. Tüm formülasyonlarda zaman faktörü $e^{-i \omega t}$ olarak alınmıştır. Makalenin kalan kısmının organizasyonu şu şekildedir. 2. Bölümde problem tanımlanmış ve ters problemin DBIM yöntemi ile sayısal olarak çözümünden bahsedilmiştir. 3. Bölümde farklı konfigürasyonlar için sayısal sonuçlar sunulmuştur. Son bölümde ise elde edilen sonuçlar özetlenmiştir.

\section{Materyal ve metod}

Problemin geometrisi Şekil 1'de verilmiştir. Ele alınan geometride uzay, $z=0$ düzlemi ile elektriksel özellikleri birbirinden farklı iki yarı sonsuz uzaya ayrılmış durumdadır. Üst yarı uzay, $\varepsilon_{1}=\varepsilon_{0}=8.854 \times 10^{-12}[\mathrm{~F} / \mathrm{m}]$ dielektrik sabiti ve $\sigma_{1}=0$ öz iletkenlik katsayısı ile karakterize edilen hava, alt yarı uzay ise $\varepsilon_{2}=\varepsilon_{r 2} \varepsilon_{0}$ dielektrik sabiti ve $\sigma_{2}$ öz iletkenlik katsayısı ile karakterize edilen kuru toprak olarak alınmıştır. Hem hava hem de kuru toprak manyetik olmayan ortamlar olup manyetik geçirgenlikleri boş uzayın manyetik geçirgenliği olan $\mu_{0}=4 \pi \times 10^{-7}[\mathrm{H} / \mathrm{m}]$ 'a eşittir.
Burada ele alınan problem, arka plan olarak adlandıracağımız bu geometrinin alt yarı uzayında bulunan olası cisimlerin geometrik ve elektriksel özelliklerini ortaya çıkartmaktır. $\mathrm{Bu}$ amaç doğrultusunda, bir görüntüleme bölgesi belirlenir ve bu bölge boyunca kompleks dielektrik sabitini belirlemek için bir ters saçılma algoritması uygulanır. Şekil üzerinde $D$ ile gösterilen bu hacimsel bölge yeniden yapılandırma bölgesi olarak da adlandırılır. Çünkü, ters problem çözümünde bu bölgenin kompleks dielektrik sabiti değeri için bir başlangıç değeri belirlenir ve iteratif olarak bu değer güncellenir yani bölge yeniden yapilandirılir.

$D$ bölgesi bir elektromanyetik dalga kaynağı ile aydınlatılır ve ölçüm bölgesi olarak adlandırılan ve şekil üzerinde $S$ ile gösterilen bir bölgede elektromanyetik alan ölçümleri yapılır. $D$ içerisinde herhangi bir cisim yoksa bu bölge elektriksel olarak alt yarı uzaya yani toprağa eşdeğer olacak ve $S$ bölgesinde ölçülen toplam alan $\mathbf{E}^{\text {top }}$, elektromanyetik dalga kaynağından doğrudan gelen alan ile $z=0$ düzlemindeki hava-toprak ara yüzeyinden yansıyan alanların toplamına eşit olacaktır. Herhangi bir gömülü cismin mevcut olmadığı durumdaki bu alan, arka plan alanı olarak adlandırılacak ve $\mathbf{E}^{b}$ ile gösterilecektir.

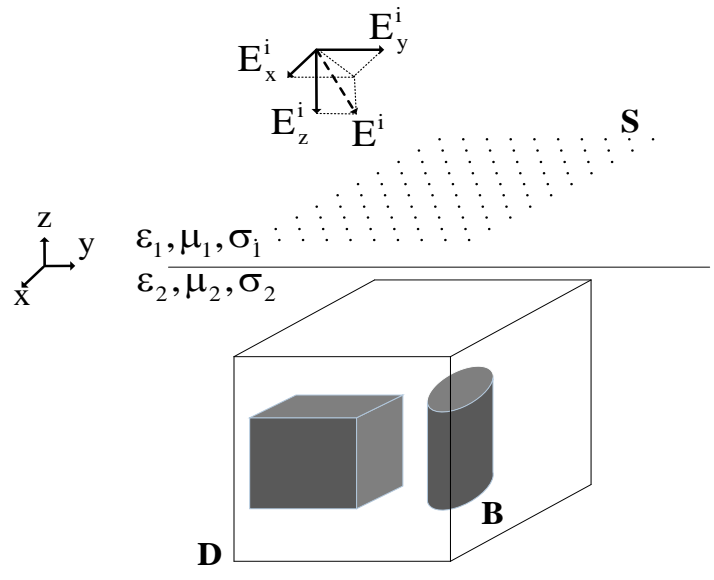

Şekil 1. Problemin geometrisi

Buna karşın, $D$ içerisinde alt yarı uzayınkinden farklı elektriksel özelliklere sahip cisimler bulunuyorsa bu durumda toplam alana, bu cisimlerden saçılan alan da katkı sağlayacaktır. Gömülü cisimlerden saçılan alan $\mathbf{E}^{S}$ ile gösterilecek olursa bu durumda toplam elektrik alan $\mathbf{E}^{\text {top }}(\mathbf{r})$,

$$
\mathbf{E}^{t o p}(\mathbf{r})=\mathbf{E}^{b}(\mathbf{r})+\mathbf{E}^{s}(\mathbf{r})
$$

olacaktır. $\mathrm{O}$ halde en genel halde herhangi bir $\mathbf{r}(x, y, z) \in$ $\mathfrak{R}^{3}$ noktasındaki toplam alan için

$$
\mathbf{E}^{t o p}(\mathbf{r})=\left\{\begin{array}{c}
\mathbf{E}^{b}(\mathbf{r}) ; D \text { içinde cisim yoksa } \\
\mathbf{E}^{b}(\mathbf{r})+\mathbf{E}^{s}(\mathbf{r}) ; D \text { içinde cisim varsa }
\end{array}\right.
$$

yazmak uygun olacaktır. Hem $\mathbf{E}^{\text {top }}(\mathbf{r})$ ve hem de $\mathbf{E}^{b}(\mathbf{r})$ homojen Helmholtz denklemini sağlayacaktır. 


$$
\begin{gathered}
\nabla^{2} \mathbf{E}^{t o p}(\mathbf{r})+k^{2}(\mathbf{r}) \mathbf{E}^{t o p}(\mathbf{r})=0 \\
\nabla^{2} \mathbf{E}^{b}(\mathbf{r})+k_{b}^{2}(\mathbf{r}) \mathbf{E}^{b}(\mathbf{r})=0
\end{gathered}
$$

Denklem (1) göz önünde bulundurularak bu iki denklemden saçılan alan için

$$
\nabla^{2} \mathbf{E}^{s}(\mathbf{r})+k_{b}^{2} \mathbf{E}^{s}(\mathbf{r})=-k_{b}^{2}\left(\frac{k^{2}(\mathbf{r})-k_{b}^{2}}{k_{b}^{2}}\right) \mathbf{E}^{t o p}(\mathbf{r})
$$

denklemi türetilir. Burada $\omega=2 \pi f$ açısal frekans olmak üzere $k_{b}^{2}=\omega^{2} \varepsilon_{r b} \varepsilon_{0} \mu_{0}+\sigma_{b} \mu_{0}$ arka plandaki dalga sayısının karesi, $\quad k^{2}(\mathbf{r})=\omega^{2} \varepsilon_{r}(\mathbf{r}) \varepsilon_{0} \mu_{0}+\sigma(\mathbf{r}) \mu_{0} \quad$ ise en genel durumda herhangi bir $\mathbf{r}(x, y, z)$ noktasındaki dalga sayısının karesidir. Denklem (5)'te eşitliğin sağ tarafında görülen parantez içindeki ifade kontrast fonksiyonu olarak adlandırılır ve $\mathbf{r}$ noktasının elektriksel olarak arka plandan ne kadar farklı olduğunun bir ölçütüdür. $\chi(\mathbf{r})$ ile gösterilecek olan kontrast fonksiyonu,

$$
\chi(\mathbf{r})=\left(\frac{k^{2}(\mathbf{r})}{k_{b}^{2}}-1\right)=\left(\frac{\varepsilon(\mathbf{r})}{\varepsilon_{b}}-1\right)
$$

ters problemin çözümüyle açığa çıkartılmak istenen büyüklüktür. Şekil 1'den de görüleceği üzere gömülü cisimlerin dışında kalan noktalarda $k(\mathbf{r})=k_{b}$ olacağından kontrast fonksiyonu sifir olacaktır. Yani $\chi(\mathbf{r})$, sadece $\mathbf{r} \in$ $B$ 'de sıfirdan farklıdır. O halde, yeniden yapılandırma sonucu elde edilen $\chi(\mathbf{r})$ 'nin $D$ 'deki dağılımı çizdirildiğinde, sıfırdan farklı gözüken yerlerin cisimlerin geometrisini belirleyeceği açıktır. Denklem (5)'teki eşitliğin sağında kaynak terimi olarak nokta kaynak fonksiyonu (Dirac delta) kullanılırsa elde edilen

$$
\nabla^{2} \overline{\mathbf{G}}\left(\mathbf{r}, \mathbf{r}^{\prime}\right)+k_{b}^{2} \overline{\mathbf{G}}\left(\mathbf{r}, \mathbf{r}^{\prime}\right)=\overline{\mathbf{I}} \delta\left(\mathbf{r}-\mathbf{r}^{\prime}\right)
$$

biçimindeki dalga denkleminin uygun sınır koşulları altında çözümü probleme 3B Green fonksiyonunu verecektir. 3B problemler söz konusu olduğunda Green fonksiyonları diyadik formda yani tensör formunda olurlar. Diyadik form, keyfi kutuplu bir kaynağın oluşturduğu tüm alan bileşenlerini yazmanın en uygun ve pratik yoludur. Denklem (7)'de verilen dalga denkleminin çözümünden elde edilecek Green fonksiyonu $\quad \overline{\mathbf{G}}=G_{x x} \hat{x} \hat{x}+G_{x y} \hat{x} \hat{y}+G_{x z} \hat{x} \hat{z}+$ $G_{y x} \hat{y} \hat{x}+G_{y y} \hat{y} \hat{y}+G_{y z} \hat{y} \hat{z}+G_{z x} \hat{z} \hat{x}+G_{z y} \hat{z} \hat{y}+G_{z z} \hat{z} \hat{z}$ formunda olup elektrik alan diyadik Green fonksiyonudur $[20,21]$. Burada $\overline{\mathbf{I}}=\hat{x} \hat{x}+\hat{y} \hat{y}+\hat{z} \hat{z}$ birim diyadik veya birim tensör olarak adlandırılır ve $\delta$, Dirac delta fonksiyonunu göstermektedir. Buna göre, Green fonksiyonunun nokta kaynağın oluşturduğu elektrik alana eşit olduğunu söyleyebiliriz. Denklem (5)'teki asıl kaynak fonksiyonu için çözüm, nokta kaynak çözümlerinin süperpozisyonu cinsinden ifade edilebilir.

Yukarıda verilen vektörel dalga denkleminin çözümünden Green fonksiyonun türetilmesi hem matematiksel ve hem de hesaplama bakımından oldukça uzun ve yorucu işlemleri gerektirir. Denklemin analitik çözümü olmayıp Green fonksiyonunun diyadik bileşenlerinin bulunması yavaş yakınsayan Sommerfeld integrallerinin sayısal çözümünü gerektir. $\mathrm{Bu}$ çalışmanın esas konusu olmadığından burada diyadik Green fonksiyonun türetilmesine yer verilmeyecektir. İki parçalı uzay diyadik Green fonksiyonun detaylı çıkarımı [22]'de verilmiştir. Denklem (5) ve (7) kullanılarak saçılan alan için

$$
\mathbf{E}^{s}(\mathbf{r})=k_{b}^{2} \int_{D} \overline{\mathbf{G}}\left(\mathbf{r}, \mathbf{r}^{\prime}\right) \chi\left(\mathbf{r}^{\prime}\right) \mathbf{E}^{t o p}\left(\mathbf{r}^{\prime}\right) d \mathbf{r}^{\prime}
$$

integral denklemi türetilir. $\mathbf{E}^{S}(\mathbf{r}), \quad D$ bölgesinin bir elektromanyetik dalga ile aydınlatılması durumunda $D$ içindeki cisimlerin $\mathbf{r}$ noktasında oluşturacağı saçılan alandır. İntegralin içinde görülen $\mathbf{E}^{t o p}\left(\mathbf{r}^{\prime}\right)$ ise $D$ içindeki toplam alandir ve bu alan

$$
\mathbf{E}^{t o p}(\mathbf{r})=\mathbf{E}^{b}(\mathbf{r})+k_{b}^{2} \int_{D} \overline{\mathbf{G}}\left(\mathbf{r}, \mathbf{r}^{\prime}\right) \chi\left(\mathbf{r}^{\prime}\right) \mathbf{E}^{t o p}\left(\mathbf{r}^{\prime}\right) d \mathbf{r}^{\prime}
$$

integral denkleminden bulunur. Denklem (8) ve (9) ters saçılma probleminin çözümünde kullanılan iki temel denklemdir. $\mathrm{Bu}$ iki denklemin sayısal çözümünden görüntüleme bölgesindeki bilinmeyen kontrast fonksiyonu belirlenir. Sayısal çözüm için $D$ bölgesi $N_{D}$ adet kübik hücreye bölünerek ayrıklaştırılır. Ölçüm bölgesinde $(S)$ toplam $N_{S}$ noktada elektrik alan ölçümü yapıldığı varsayılırsa yukarıdaki integraller ayrık formda,

$$
\begin{gathered}
\sum_{i=1}^{\mathrm{N}_{\mathrm{s}}} \mathbf{E}^{S}\left(\mathrm{r}_{i}\right)=k_{b}^{2} \sum_{i=1}^{\mathrm{N}_{S}} \sum_{j=1}^{\mathrm{N}_{D}} \overline{\mathbf{G}}\left(\mathbf{r}_{i}, \mathbf{r}_{j}\right) \chi\left(\mathbf{r}_{j}\right) \mathbf{E}^{t o p}\left(\mathbf{r}_{j}\right) \Delta \mathrm{v}_{j} ; \\
\mathbf{r}_{i} \in S ; \mathbf{r}_{j} \in D ; i=1,2, . . N_{S} ; j=1,2, \ldots N_{D}
\end{gathered}
$$

ve

$$
\begin{gathered}
\sum_{i=1}^{\mathrm{N}_{D}} \mathbf{E}^{t o p}\left(\mathrm{r}_{i}\right)-k_{b}^{2} \sum_{i=1}^{\mathrm{N}_{D}} \sum_{j=1}^{\mathrm{N}_{D}} \overline{\mathbf{G}}\left(\mathbf{r}_{i}, \mathbf{r}_{j}\right) \chi\left(\mathbf{r}_{j}\right) \mathbf{E}^{t o p}\left(\mathbf{r}_{j}\right) \Delta \mathrm{v}_{j} \\
=\sum_{i=1}^{\mathrm{N}_{D}} \mathbf{E}^{b}\left(\mathrm{r}_{i}\right) \\
\mathbf{r}_{i} \in D ; \mathbf{r}_{j} \in D ; i=1,2, . . N_{D} ; j=1,2, \ldots N_{D}
\end{gathered}
$$

olarak yazılabilir. Matris denkleme dönüştürülmüş bu denklemler daha kompakt bir formda,

$$
\overline{\mathbf{E}}^{s}=\overline{\overline{\mathbf{G}}}^{s} \operatorname{diag}(\overline{\mathbf{X}}) \overline{\mathbf{E}}^{\text {top }}
$$

ve

$$
\overline{\mathbf{E}}^{t o p}=\left(\overline{\overline{\mathbf{I}}}-\overline{\overline{\mathbf{G}}}^{D} \operatorname{diag}(\overline{\mathbf{X}})\right)^{-1} \overline{\mathbf{E}}^{b}
$$

olarak yazılabilir. Burada $\overline{\overline{\mathbf{I}}}, 3 N_{D} \times 3 N_{D}$ boyutunda birim matristir. $\overline{\mathbf{E}}^{s}, 3 N_{S} \times 1$ boyutunda bir vektör $\overline{\mathbf{E}}^{t o p}$ ve $\overline{\mathbf{E}}^{b}$ ise $3 N_{D} \times 1$ boyutunda vektörlerdir. $\overline{\overline{\mathbf{G}}}{ }^{S}, D$ içindeki her bir noktadan ölçüm bölgesi $S$ 'deki noktalara tanımlı Green 
fonksiyonu katsayılarından oluşan $3 N_{S} \times 3 N_{D}$ boyutunda bir matristir. $\overline{\overline{\mathbf{G}}}^{D}$ ise $D$ 'deki noktaların kendi içinde tanımlı Green katsayılarından oluşan $3 N_{D} \times 3 N_{D}$ boyutunda bir matristir. Bu matrisler,

$$
\overline{\overline{\mathbf{G}}}^{S \text { veya } D}=k_{b}^{2} \int_{D} \overline{\mathbf{G}}\left(\mathbf{r}, \mathbf{r}^{\prime}\right) d \mathbf{r}^{\prime} ; \mathbf{r} \in S \text { veya } D
$$

integralinin sayısal hesabından bulunurlar. $\operatorname{diag}($.$) bir$ vektörü diyagonal bir matrise dönüştüren operatördür.

$S$ 'de ölçülen saçılan verilerini kullanarak $D$ 'deki kontrast fonksiyonu dağılımını belirlemekten ibaret olan ters saçılma problemi kötü kurulmuş bir problem olup Denklem (12)'den $\overline{\mathbf{X}}$ için uygun bir çözümünün bulunabilmesi için regülerizasyon gereklidir. $\mathrm{Bu}$ amaçla Tikhonov regülerizasyonu uygulanmıştır. Tikhonov regülerizasyon yönteminde,

$$
f(\overline{\mathbf{X}})=\left\|\left(\overline{\overline{\mathbf{G}}}^{s} \overline{\mathbf{E}}\right)-\overline{\mathbf{E}}^{s}\right\|^{2}+\alpha\|\overline{\mathbf{X}}\|^{2}
$$

fonksiyonelini minimize edecek $\overline{\mathbf{X}}$ aranır. Bu ifadede görülen $\alpha$ yeterince küçük pozitif bir sayı olup regülerizasyon parametresi olarak adlandırılır. Buradan $\overline{\mathbf{X}}$ için

$$
\overline{\mathbf{X}}=\left[\left(\overline{\overline{\mathbf{G}}}^{S}\right)^{H} \overline{\overline{\mathbf{G}}}^{S}+\alpha \overline{\overline{\mathbf{I}}}\right]^{-1}\left(\overline{\overline{\mathbf{G}}}^{S}\right)^{H} \overline{\mathbf{E}}^{S}
$$

çözümü elde edilir. Denklemde görülen $H$ Hermisyen anlamına gelmektedir. Bir sonraki bölümde burada tanımlanan ters saçılma probleminin bozulmuş born iteratif yöntemi ile çözümünden bahsedilecektir.

\subsection{Bozulmuş born iteratif yöntemi (DBIM)}

Bu yöntemde saçlan alan verisinden kontrast fonksiyonu $\overline{\mathbf{X}}$ 'yı elde etmek için iteratif (yinelemeli) bir ters saçılma algoritması kullanılır. Kontrast fonksiyonunun başlangıç değeri $\overline{\mathbf{X}}^{0}$, Born yaklaşımı kullanılarak elde edilir. Bu yaklaşımında cisimlerden saçılan alan ihmal edilerek toplam alanın arka plan alanına eşit olduğu yani $\mathbf{E}_{q}^{\text {top }}\left(\mathbf{r}^{\prime}\right) \approx \mathbf{E}_{q}^{b}\left(\mathbf{r}^{\prime}\right)$ kabul edilir. $\mathrm{Bu}$ kabul, yeniden yapılandırma bölgesinin başlangıç dielektrik özelliklerinin arka plan ile aynı alınması anlamına gelir. Buna göre $\overline{\mathbf{X}}^{0}$,

$$
\overline{\mathbf{E}}^{s}=\overline{\overline{\mathbf{G}}}^{s} \operatorname{diag}\left(\overline{\mathbf{X}}^{0}\right) \overline{\mathbf{E}}^{b}
$$

denkleminden Denklem (16)'da verilen Tikhonov regülerizasyonu uygulanarak hesaplanır. Bulunan cisim fonksiyonu, Denklem (13)'te yerine yazılır ve böylece yeniden yapılandırma bölgesindeki toplam alan düz problem çözümünden bulunarak güncellenir.

DBIM algoritmasında iterasyonun başlangıcında görüntüleme bölgesinin dielektrik geçirgenliği arka planınki ile aynı kabul edilmekte ve her iterasyonda güncellenmektedir. Benzer olarak, Green fonksiyonu da başlangıçta arka plan Green fonksiyonu $\overline{\mathbf{G}}$ ile aynı alınmakta ve her iterasyonda güncellenmektir. $\overline{\mathbf{G}}^{g}$ ile gösterilecek olan güncellenen Green fonksiyonu,

$$
\begin{aligned}
& \overline{\mathbf{G}}^{g}\left(\mathbf{r}, \mathbf{r}^{\prime}\right) \\
&=\overline{\mathbf{G}}\left(\mathbf{r}, \mathbf{r}^{\prime}\right)+k_{b}^{2} \int_{D} \overline{\mathbf{G}}\left(\mathbf{r}, \mathbf{r}^{\prime \prime}\right) \chi\left(\mathbf{r}^{\prime \prime}\right) \overline{\mathbf{G}}^{g}\left(\mathbf{r}^{\prime \prime}, \mathbf{r}^{\prime}\right) d \mathbf{r}^{\prime \prime} \\
& \quad \mathbf{r} \in S, \mathbf{r}^{\prime} \in D, \mathbf{r}^{\prime \prime} \in D
\end{aligned}
$$

integral denkleminin çözümünden bulunacaktır. Green fonksiyonunu güncellemek için gözlem noktalarına konumlandırılmış noktasal kaynağın yeniden yapılandırma bölgesinde meydana getirdiği toplam alan hesaplanır. $\mathrm{Bu}$ denklem kompakt formda

$$
\overline{\overline{\mathbf{G}}}^{S g}=\left[\left(\overline{\overline{\mathbf{I}}}-\overline{\overline{\mathbf{G}}}^{D} \operatorname{diag}(\overline{\mathbf{X}})\right)^{-1}\left(\overline{\overline{\mathbf{G}}}^{S}\right)^{T}\right]^{T}
$$

biçiminde yazılabilir. Burada $T$ transpozu göstermektedir ve $\overline{\overline{\mathbf{G}}}^{s g}$ ayrık formdaki güncel Green fonksiyonu matrisidir.

Yöntemin bir sonraki adımında güncel kontrast fonksiyonu, toplam alan ve Green fonksiyonu Denklem (8)'de kullanılarak,

$$
\mathbf{E}^{s h}(\mathbf{r})=k_{b}^{2} \int_{D} \overline{\mathbf{G}}^{s g}\left(\mathbf{r}, \mathbf{r}^{\prime}\right) \chi\left(\mathbf{r}^{\prime}\right) \mathbf{E}^{t o p}\left(\mathbf{r}^{\prime}\right) d \mathbf{r}^{\prime}
$$

ölçüm noktalarındaki saçılan alan hesaplanır. Hesaplama sonucu elde edilen saçılan alan $\mathbf{E}^{\text {sh }}$ ile gösterilmektedir. Ölçülerek elde edilen gerçek saçılan alan verisi ile hesap yoluyla elde edilen saçılan alan arasındaki fark saçılan alan,

$$
\partial \mathbf{E}^{s}(\mathbf{r})=\mathbf{E}^{s}(\mathbf{r})-\mathbf{E}^{s h}(\mathbf{r})
$$

Denklem (8)'de kullanılırsa fark saçılan alan için

$$
\partial \mathbf{E}^{S}(\mathbf{r})=k_{2}^{2} \int_{D} \overline{\mathbf{G}}^{s g}\left(\mathbf{r}, \mathbf{r}^{\prime}\right) \partial \chi\left(\mathbf{r}^{\prime}\right) \mathbf{E}^{t o p}\left(\mathbf{r}^{\prime}\right) d \mathbf{r}^{\prime}
$$

integral denklemi elde edilir. Bu denklem ise kompakt formda,

$$
\partial \overline{\mathbf{E}}^{s}=\overline{\overline{\mathbf{G}}}^{s g} \operatorname{diag}(\partial \overline{\mathbf{X}}) \overline{\mathbf{E}}^{t o p}
$$

olarak yazilır. Buradan hesaplanan fark cisim fonksiyonu bir önceki iterasyon adımındaki cisim fonksiyonuna eklenerek

$$
\overline{\mathbf{X}}_{n+1}=\overline{\mathbf{X}}_{n}+\partial \overline{\mathbf{X}}_{n}
$$

şeklinde kontrast fonksiyonu güncellenir ve

$$
\text { Hata }=\frac{\sum_{m=1}^{N_{s}}\left|\mathbf{E}^{s}\left(\mathbf{r}_{m}\right)-\mathbf{E}_{n}^{s h}\left(\mathbf{r}_{m}\right)\right|^{2}}{\sum_{m=1}^{N_{s}}\left|\mathbf{E}^{s}\left(\mathbf{r}_{m}\right)\right|^{2}} \leq \epsilon
$$

ile tanımlanan bağıl hata değeri önceden belirlenen küçük bir $\epsilon$ değerine veya iterasyon sayısı $n$ başlangıçta belirlenen bir maksimum iterasyon sayısı $N_{\text {max }}$ 'a ulaşıncaya kadar iterasyona devam edilir. Bu ifadede görülen $\mathbf{E}^{s}\left(\mathbf{r}_{m}\right)$, ölçüm bölgesi üzerindeki $\mathbf{r}_{m}$ noktasında ölçülen saçılan alan değeri, 
$\mathbf{E}_{n}^{s h}\left(\mathbf{r}_{m}\right)$ ise $n$ 'yinci iterasyon adımında ölçüm bölgesi üzerindeki $\mathbf{r}_{m}$ noktasında hesaplanan saçılan alan değeridir. $q=1,2, \ldots, N_{q}$ geliş açısı sayısını göstermek üzere toplam $N_{q}$ farklı geliş açısı için aynı prosedür uygulanır ve her bir açı için elde edilen kontrast fonksiyonlarının ortalaması alınarak sonuç kontrast fonksiyonu,

$$
\overline{\mathbf{X}}=\frac{\sum_{q=1}^{N_{q}} \overline{\mathbf{X}}_{q}}{N_{q}}
$$

ifadesinden elde edilir. Herhangi bir geliş açısı için DBIM algoritmasına ait işlem adımları şu şekilde özetlenebilir.

DBIM algoritması:

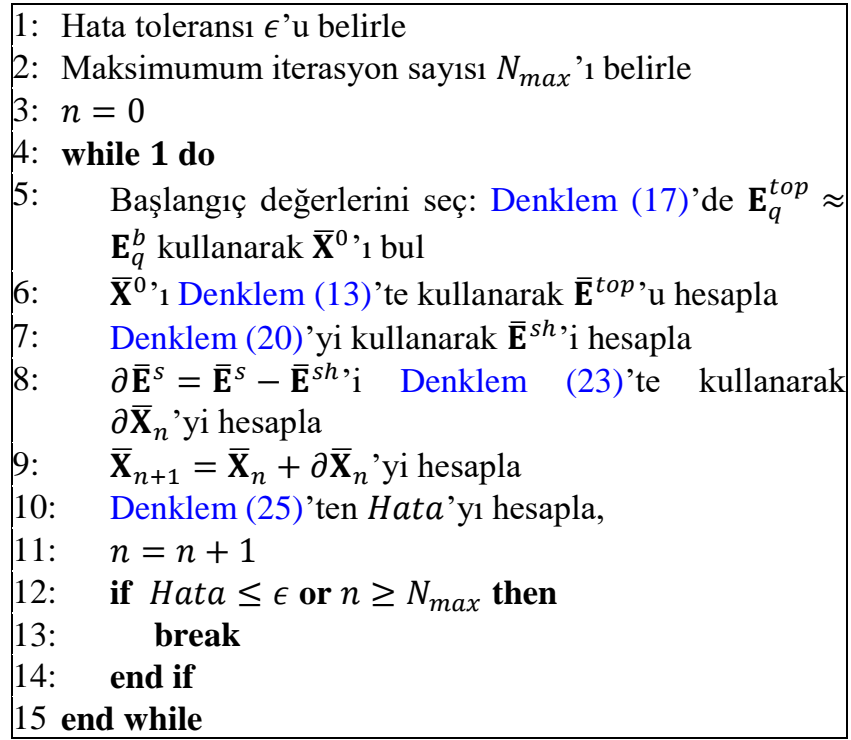

\section{Bulgular ve tartışma}

$\mathrm{Bu}$ bölümde DBIM kullanılarak yer altına gömülü $3 \mathrm{~B}$ cisimlerin yeniden yapılandırmasına ilişkin sayısal örnekler sunulmuştur. Tüm örneklerde çalışma frekansı $300 \mathrm{MHz}$ olarak alınmıştır. Üst yarı uzay hava $\left(\varepsilon_{1}=\varepsilon_{r 1}, \sigma_{1}=0\right)$, alt yar1 uzay ise parametreleri ise $\varepsilon_{2}=(2 \sim 4) \varepsilon_{0}$ ve $\sigma_{2}=$ $10^{-4} \sim 10^{-6}[S / m]$ [23] civarında olan kuru toprak olarak belirlenmiştir. Yeniden yapılandırma bölgesi 100 farklı geliş açısında düzlem dalga ile ardışık olarak aydınlatılmış ve saçılan alanlar ara yüzeyden $0,2 m$ yukarıda, 200 farklı noktada, ardışık iki nokta arası $0.1 \mathrm{~m}$ olacak şekilde düzlemsel bir bölgede ölçülmüştür. İterasyon durdurma kriteri $\epsilon=10^{-6}$ alınmıştır. Elektrik alan ölçüm verileri düz problemin moment yöntemi ile çözümünden elde edilen sentetik verilerdir. $D$ bölgesindeki düzlemsel kesitler boyunca bağıl dielektrik geçirgenlik $\varepsilon_{r}(\mathrm{r})$ ve iletkenlik $\sigma(\mathrm{r})$ dağılımları çizdirilmiştir. Ters problem çözümüyle yeniden yapılandırma bölgesindeki $\chi(\mathrm{r})$ dağılımı elde edildikten sonra bu bölgedeki bağıl dielektrik geçirgenlik ve iletkenlik değişimi Denklem (6) kullanılarak sırasıyla,

$$
\varepsilon_{r}(\mathrm{r})=\operatorname{real}\left[(\chi(\mathrm{r})+1) \varepsilon_{r b}\right]
$$

$$
\sigma(\mathrm{r})=\operatorname{imag}\left[(\chi(\mathrm{r})+1) \varepsilon_{b}\right] \omega
$$

denklemlerinden bulunur.

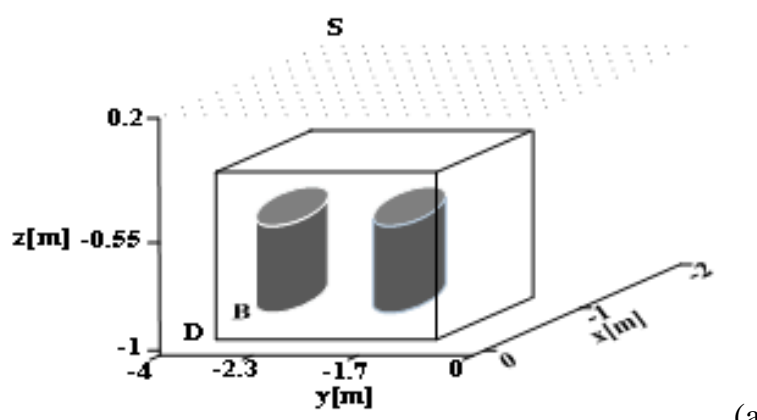

(a)

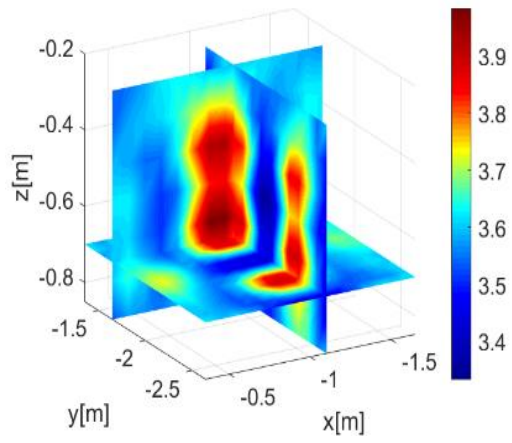

(b)

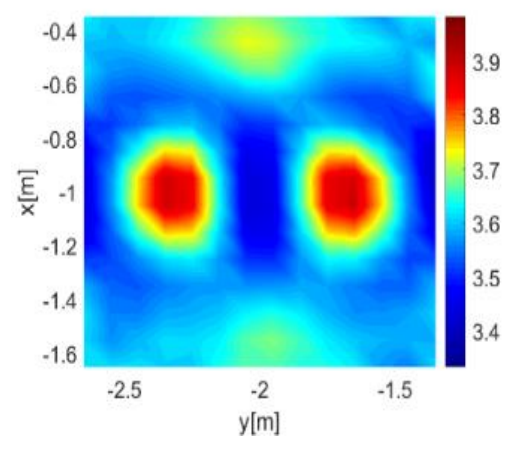

(c)

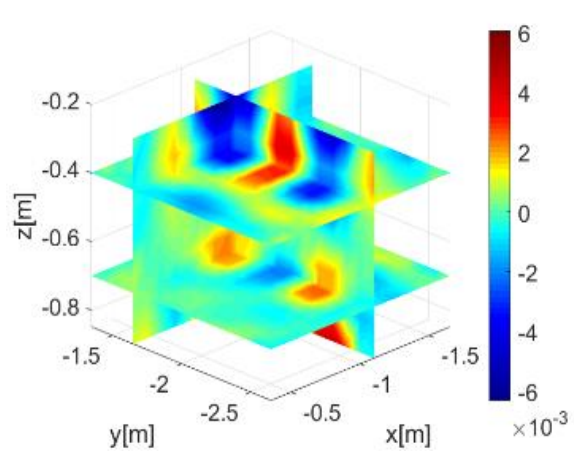

Şekil. 2. $\varepsilon_{r B}=4.5$, iletkenliği $\sigma_{B}=10^{-3}(\mathrm{~S} / \mathrm{m})$ olan iki silindirik cismin yeniden yapılandırması (a) orijinal geometri (b) $x=-1.1, \quad y=-1.3, \quad z=-0.7$ $\begin{array}{llll}\text { düzlemlerindeki } & \varepsilon_{r}(\mathrm{r}) \text { dağılımı } & \text { (c) } \mathrm{y}=-0.7\end{array}$ düzlemindeki $\varepsilon_{r}(\mathrm{r})$ dağılımı (d) $x=-1.1, y=-1.3, z=$ -0.7 düzlemlerindeki $\sigma(\mathrm{r})$ dağılımı $\quad$ (e) $\mathrm{y}=-0.7$ düzlemindeki $\sigma(\mathrm{r})$ dağılımı 


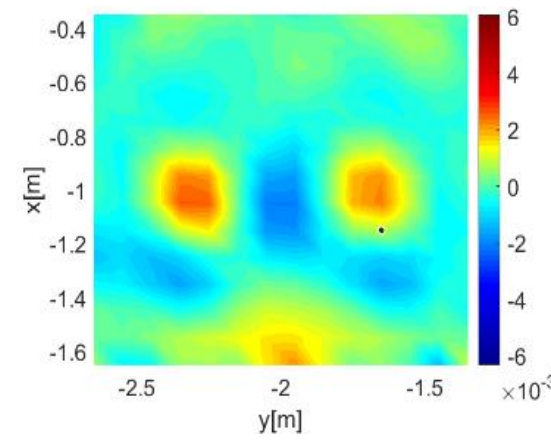

(e)

Şekil. 2.(devam) $\varepsilon_{r B}=4.5$, iletkenliği $\sigma_{B}=10^{-3}(S /$ $m$ ) olan iki silindirik cismin yeniden yapılandırmas1 (a) orijinal geometri (b) $x=-1.1, y=-1.3, z=-0.7$ $\begin{array}{llll}\text { düzlemlerindeki } & \varepsilon_{r}(\mathrm{r}) \text { dağılımı } & \text { (c) } \mathrm{y}=-0.7\end{array}$ düzlemindeki $\varepsilon_{r}(\mathrm{r})$ dağılımı (d) $x=-1.1, y=-1.3, z=$ -0.7 düzlemlerindeki $\sigma(\mathrm{r})$ dağılımı $\quad$ (e) $\mathrm{y}=-0.7$ düzlemindeki $\sigma(\mathrm{r})$ dağılımı

İlk örnekte kuru toprağın elektriksel parametreleri $\varepsilon_{2}=$ $3,3 \varepsilon_{0}$ ve $\sigma_{2}=10^{-6}(\mathrm{~S} / \mathrm{m})$ olarak alınmış ve yükseklikleri $0.7 \mathrm{~m}$ ve yarıçapları $0.2 \mathrm{~m}$ olan iki silindirik cismin bu ortama gömülü olduğu varsayılmıştır. Silindirik cisimlerin merkez koordinatlar1 $(-1,-1.7,-0.55)$ ve $(-1,2.3,-0.55)$ 'dir. Cisimlerin her ikisinin de bağıl dielektrik geçirgenliği $\varepsilon_{B}=$ $4.5 \varepsilon_{0}$, iletkenlik katsayısı $\sigma_{B}=10^{-3}(\mathrm{~S} / \mathrm{m})$ 'dir. Yeniden yapılandırma bölgesinin merkezi $(-1,-2,-0.55)$ ve boyutları $1.4 \mathrm{~m} \times 1.4 \mathrm{~m} \times 0.7 \mathrm{~m}$ olarak alınmıştır. $\mathrm{Bu}$ konfigürasyon için DBIM algoritması kullanılarak elde edilen yeniden yapılandırma sonuçları Şekil 2'de verilmiştir. Şekil 2a'da ele alınan örneğe ait orijinal geometri verilmiştir. Şekil 2b ve c'de yeniden yapılandırma bölgesindeki elde edilen bağıl dielektrik geçirgenlik dağılımının farklı kesit düzlemlerindeki çizimleri görülmektedir. Şekil $2 d$ ve e'de ise aynı düzlemlerdeki iletkenlik dağılımı görülmektedir. Bu şekillerden hem cismin geometrisi hem de dielektrik özelliklerinin yüksek doğrulukla elde edildiği görülmektedir.

$\mathbf{s}$

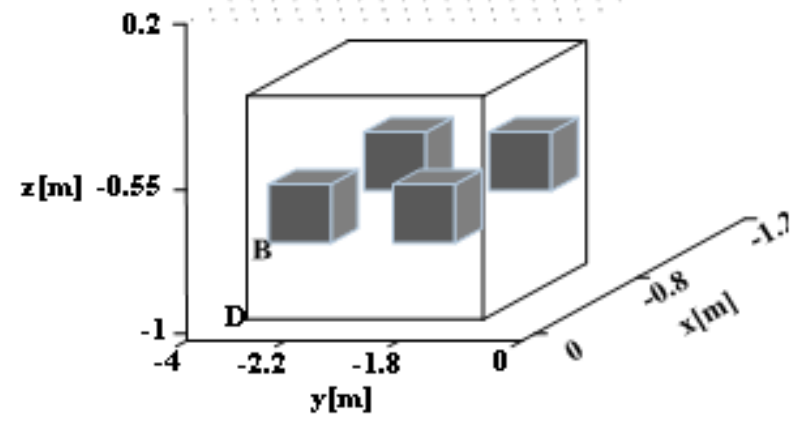

(a)

Şekil 3. $\varepsilon_{r B}=4.5, \sigma_{B}=10^{-3}(S / m)$ olan dört kübik cisim yeniden yapılandırmas1 (a) orijinal geometri (b) $x=$ $-0.7, y=-2.2, z=-0.55$ düzlemlerindeki $\quad \varepsilon_{r}(\mathrm{r})$ dağılımı (c) $\mathrm{y}=-0.55$ düzlemindeki $\varepsilon_{r}(\mathrm{r})$ dağılımı (d) $x=-0.7, y=-2.2, z=-0.7$ düzlemlerindeki $\sigma(\mathrm{r})$ dağılımı (e) $\mathrm{y}=-0.55$ düzlemindeki $\sigma(\mathrm{r})$ dağılımı

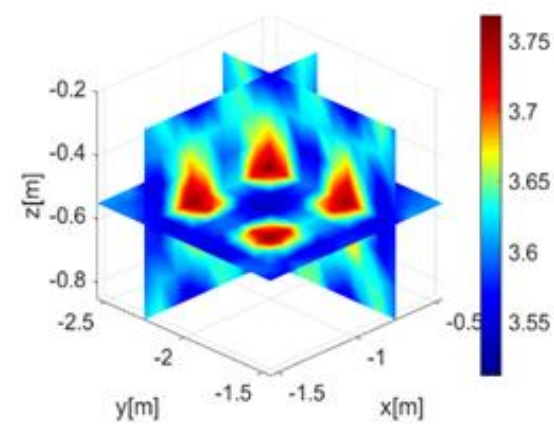

(b)

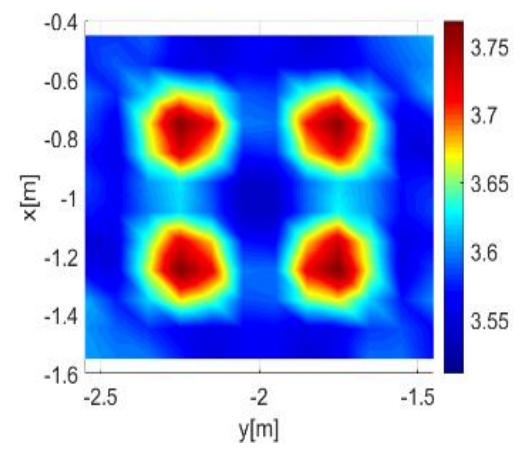

(c)
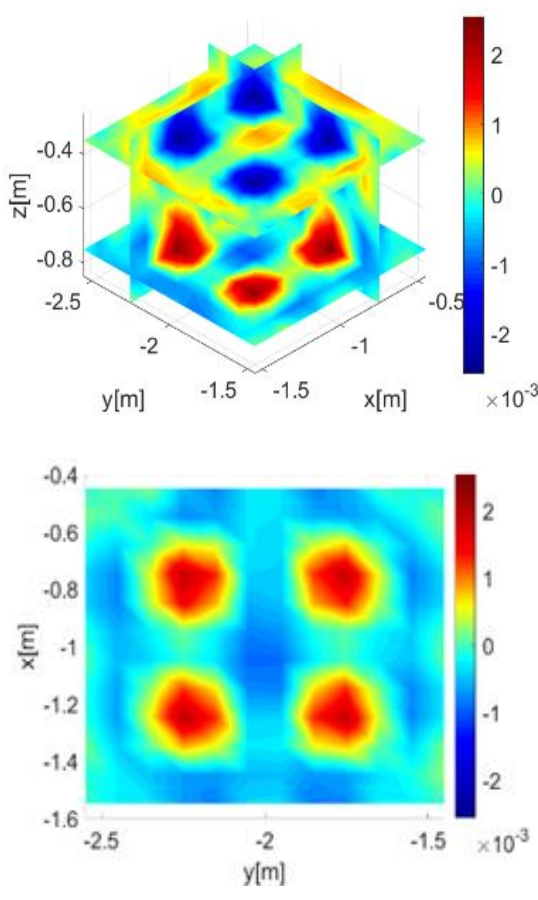

(e)

Şekil 3.(devam) $\varepsilon_{r B}=4.5, \sigma_{B}=10^{-3}(S / m)$ olan dört kübik cisim yeniden yapılandırması (a) orijinal geometri (b) $\quad x=-0.7, y=-2.2, z=-0.55$ düzlemlerindeki $\varepsilon_{r}(\mathrm{r})$ dağılımı (c) $\mathrm{y}=-0.55$ düzlemindeki $\varepsilon_{r}(\mathrm{r})$ dağllımı (d) $\quad x=-0.7, y=-2.2, z=$ -0.7 düzlemlerindeki $\sigma(\mathrm{r})$ dağılımı (e) $\mathrm{y}=-0.55$ düzlemindeki $\sigma(\mathrm{r})$ dağılımı

İkinci örnekte de kuru toprağın elektriksel parametreleri ve gömülü cisimlerin elektriksel parametreleri bir önceki örnekle aynı alınmıştır. Ancak bu kez gömülü cisimler bir ayrıtının uzunluğu $0.2 m$ olan dört tane küpten oluşmaktadır. Cisimlerin merkez noktaları $(-1.2,-1.8,-0.55)$, 
$(-1.2,-2.2,-0.55), \quad(-0.8,-1.8,-0.55) \quad$ ve $(-0.8,-2.2,-0.55)$ 'dir. Merkezi $(-1,-2,-0.55)$ noktasinda bulunan $1.2 \mathrm{~m} \times 1.2 \mathrm{~m} \times 0.7 \mathrm{~m}$ 'lik hacimsel bir bölge yeniden yapılandırma bölgesi olarak kullanılmıştır. Elde edilen yeniden yapılandırma sonuçları Şekil 3'te verilmiştir. Yine bu örnek için elde edilen sonuçlar incelendiğinde hem cisimlerin bağıl geçirgenlik hem de iletkenlik değerlerinin başarılı bir şekilde elde edilebildiği görülmektedir.
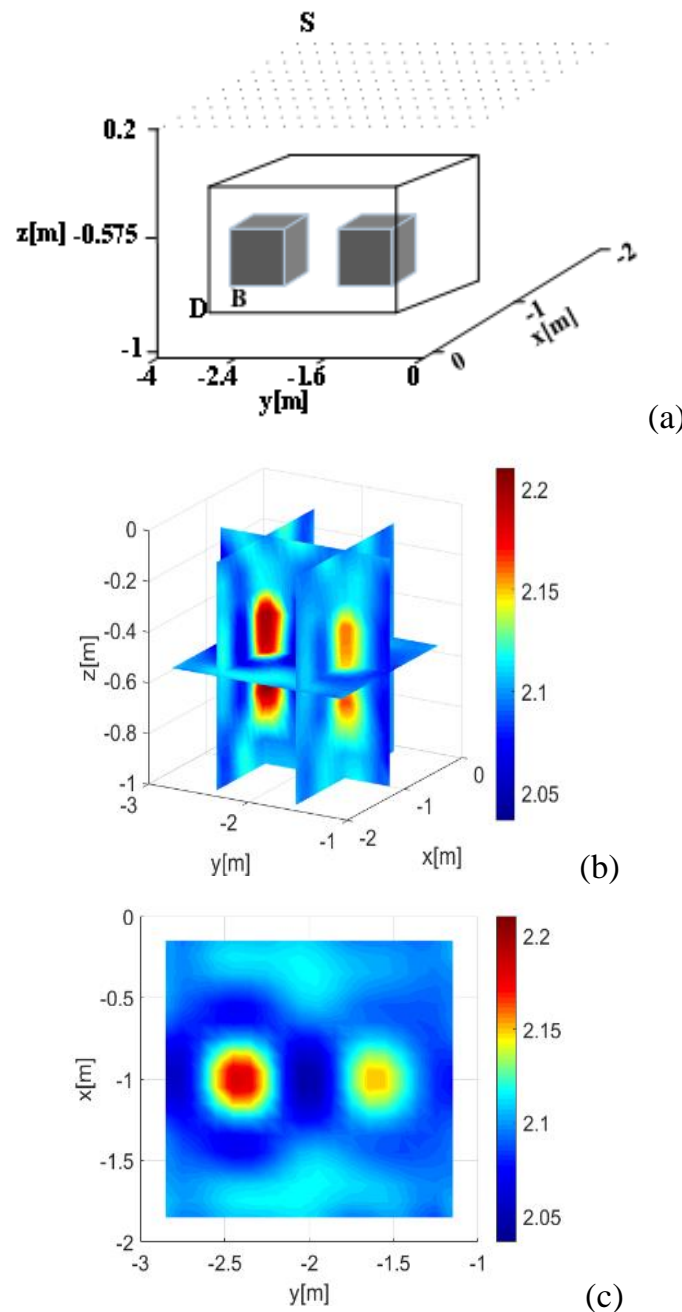

(b)

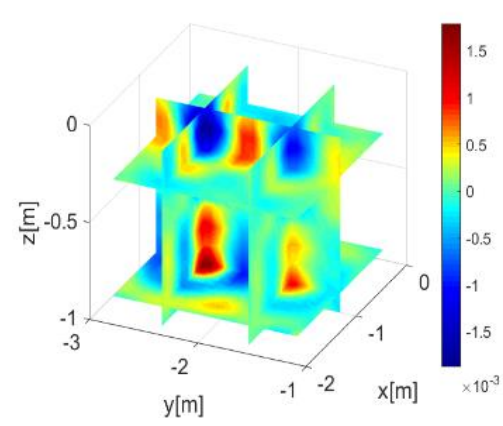

(c)

Şekil 4. $\varepsilon_{r B 1}=2.5$ ve $\varepsilon_{r B 1}=2.3, \sigma_{B}=10^{-3}(\mathrm{~S} / \mathrm{m})$ olan iki kübik cismin yeniden yapılandırması (a) orijinal geometri (b) farklı kesitlerdeki $\varepsilon_{r}(\mathrm{r})$ dağılımı (c) $\mathrm{y}=$ -0.5 düzlemindeki $\varepsilon_{r}(\mathrm{r})$ dağılımı (d) farklı kesitlerdeki $\sigma(\mathrm{r})$ dağ̊lımı (e) $\mathrm{y}=-0.7$ düzlemindeki $\sigma(\mathrm{r})$ dağ̊lımı

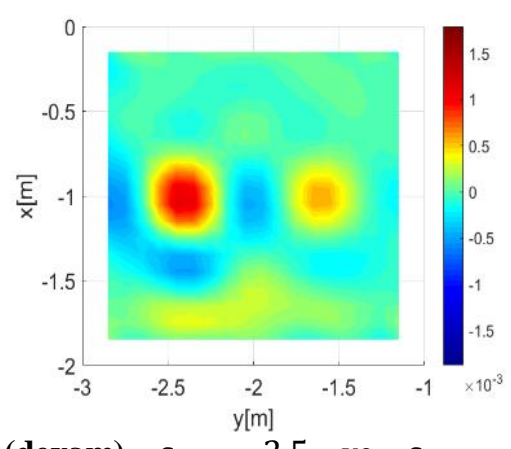

Şekil 4.(devam) $\varepsilon_{r B 1}=2.5$ ve $\varepsilon_{r B 1}=2.3, \quad \sigma_{B}=$ $10^{-3}(\mathrm{~S} / \mathrm{m})$ olan iki kübik cismin yeniden yapılandırması (a) orijinal geometri (b) farklı kesitlerdeki $\varepsilon_{r}(\mathrm{r})$ dağılımı (c) $\mathrm{y}=-0.5$ düzlemindeki $\varepsilon_{r}(\mathrm{r})$ dağılımı (d) farklı kesitlerdeki $\sigma(\mathrm{r})$ dağılımı (e) $\mathrm{y}=-0.7$ düzlemindeki $\sigma(\mathrm{r})$ dağglımı

Son örnekte elektriksel özellikleri birbirinden farklı iki cismin yeniden yapılandırması ele alınmıştır. Gömülü cisimlerin her ikisi de geometrik olarak birbirine özdeş olup bir ayrıtının uzunluğu $0.4 \mathrm{~m}$ olan küp biçimindedirler. Buna karşın, birinci cismin dielektrik geçirgenliği $\varepsilon_{B 1}=2.5 \varepsilon_{0}$ iken ikinci cisminki $\varepsilon_{B 2}=2.3 \varepsilon_{0}$ 'dır. Her iki cismin, öz iletkenlik katsayısı eşit olup $\sigma_{B 1}=\sigma_{B 2}=10^{-3} \mathrm{~S} / \mathrm{m}$ 'dir. Örneğe ait yeniden yapılandırma sonuçları Şekil 4'te sunulmuştur. Şekil $4 \mathrm{~b}$ ve c yeniden yapılandırma bölgesinde farklı kesitlerde çizdirilen bağıl dielektrik geçirgenliği göstermektedir. Yeniden yapılandırma sonuçlarına bakıldığında beklenildiği üzere, yüksek dielektrik geçirgenlikli soldaki cisim sağdakine göre daha belirgin ve daha yüksek dielektrik geçirgenlik değerine sahip olduğu anlaşılmaktadır. Aynı kesitlerde çizdirilen iletkenlik değişimleri ise Şekil $4 d$ ve e'de verilmiştir. Cisimlerin iletkenlik değerleri eşit olmasına karşın yeniden yapılandırma sonuçlarında dielektrik geçirgenlik değeri yüksek olan cismin iletkenlik değerinin de yüksek değerde elde edildiği görülmektedir. Bunun nedeni, bağıl dielektrik geçirgenlik ve iletkenliğin ters problem çözümüyle doğrudan elde edilen büyüklükler olmamasıdır. $\mathrm{Bu}$ parametreler yeniden yapılandırılan $\chi(\mathbf{r})$ 'nin Denklem (27) ve (28) kullanılmasıyla elde edilirler. Dolayısıyla, cisimlerin orijinal $\varepsilon_{r B}$ değerleri sadece yeniden yapılandırılan $\varepsilon_{r}(\mathbf{r})$ değerlerini değil $\sigma(\mathbf{r})$ değerlerini de etkilemektedir.

\section{Sonuçlar}

Bu çalışmada, yer altına gömülü üç boyutlu cisimlerin yeniden yapılandırması yani geometrik ve elektriksel özelliklerinin dışarıdan yapılan ölçümlerle ortaya çıkartılması için bozulmuş born iteratif yöntemi (Distorted Born Iterative Method-DBIM) kullanılmıștır. Problem, cisimlerin üç boyutlu ve yer altına gömülü olması nedeniyle matematiksel ve hesaplama açısından zor bir problemdir. Elde edilen sayısal sonuçlar, DBIM yönteminin düşük ve orta kontrasta sahip gömülü cisimlerin yeniden yapılandırmasında oldukça başarılı olduğunu göstermektedir. Yüksek kontrastlı cisimlerin konumları ve geometrileri başarıyla bulunurken elektriksel parametreleri olması gerekenden daha düşük değerlerde elde edilmiştir. 
Çoklu frekans tekniği ve/veya orijinal DBIM algoritmasında yapılacak değişim ve iyileştirmelerle yöntemin başarısının artırılabileceği öngörülmektedir.

\section{Çıkar çatışması}

Yazarlar çıkar çatışması olmadığını beyan etmektedir.

Benzerlik oranı (iThenticate): $\% 5$

\section{Kaynaklar}

[1] T. Durukan, R. E. Akdogan ve Y. Altuncu, Varyasyonel Born Iteratif yöntemi ile gömülü silindirik cisimlerin tespit edilmesi.2018 ELECO 2018 ElektrikElektronik ve Biyomedikal Mühendisliği Konferansı, pp 414-418 Bursa, 2018.

[2] Y. Altuncu, T. Durukan ve R. E. Akdogan, Reconstruction of two-dimensional objects buried into three-part space with locally rough interfaces via Distorted Born Iterative method. PIER, 23-41, 2019, doi: 10.2528/PIER19072203.

[3] Y. Altuncu, A numerical method of electromagnetic scattering by 3-D dielectric objects buried under 2-D locally rough surfaces, IEEE Trans Antennas Propagat., 3634-3643, $2015 . \quad$ doi: 10.1109/TAP.2015.2438859

[4] R. E. Akdogan and Y. Altuncu, Reconstruction of 3D objects buried under into half-space by using Variational Born Iterative method. 2019 23rd International Conference on Applied Electromagnetics and Communications (ICECOM), pp 1-4, Dubrovnik, Croatia, 2019.

[5] R. Pierri and G. Leone, Inverse scattering of dielectric cylinders by a second-order Born approximation, IEEE Trans. Geosci. Remote Sensing, 37, 1, 374-382, 1999. doi: 10.1109/36.739072.

[6] R. Lavarello and M. Oelze, A study on the reconstruction of moderate contrast targets using the distorted born iterative method, IEEE Trans. Ultrason., Ferroelect., 55, 1, 112-124, 2008. doi: 10.1109/TUFFC.2008.621.

[7] A. J. Hesford and W. C. Chew, Fast inverse scattering solutions using the distorted Born iterative method and the multilevel fast multipole algorithm, The Journal of the Acoustical Society of America, 128, 2, 679-690, 2010. doi: 10.1121/1.3458856.

[8] H. Tu, W, Chien, C. C. Chiu and T. M. Hu, Comparison of two different shape descriptions in the half-space inverse problem, SBMO/IEEE MTT-S International Conference on Microwave and Optoelectronics, pp 158-161 2005. Brasil, 2005. doi: 10.1109/IMOC.2005.1579985.

[9] S. F. Mahmoud, S. M. Ali and J. R. Wait, Electromagnetic scattering from a buried cylindrical inhomogeneity inside a lossy earth, Radio Sci., 16, 6, 1285-1298, 1981. doi: 10.1029/RS016i006p01285.

[10] F. Delbary, K. Erhard, R. Kress, R. Potthast ve J. Schulz, Inverse electromagnetic scattering in a twolayered medium with an application to mine detection.
Inverse Problems, 24, 1, 015002, 2008. doi: 10.1088/ 0266-5611/24/1/015002.

[11] J. Hadamard, Lectures on chauchy's Problem in linear Partial Differential Equations, Yale University Press, New Haven, 1923.

[12] Y. Liu and L. R. Ciric, An improved iterative method for inverse scattering, Proceedings of IEEE Antennas and Propagation Society International Symposium and URSI National Radio Science Meeting, pp 1656-1659 Seattle, USA, 1994. doi: 10.1109/APS.1994.408133.

[13] X. Chen, Computational methods for electromagnetic inverse scattering, John Wiley \& Sons Singapore Pte. Ltd. 2018.

[14] W. C. Chew and Y. M. Wang, Reconstruction of twodimensional permittivity distribution using the distorted Born iterative method. IEEE Trans. Med. Imaging, 9, 218-225, 1990. doi: 10.1109/42.56334.

[15] F. Li, Q. H. Liu and L.-P. Song, Three-dimensional reconstruction of objects buried in layered media using Born and Distorted Born Iterative Methods, IEEE Geosci. Remote Sensing Lett., 1, 2, 107-111, 2004. doi: 10.1109/LGRS.2004.826562.

[16] Y. Altuncu, F. Akleman, O. Semerci and C. Ozlem, Imaging of dielectric objects buried under a rough surface via distorted born iterative method, J. Phys.: Conf. Ser., 2008, doi: 10.1088/17426596/135/1/012006.

[17] N. Zaiping, Y. Feng, Z. Yanwen and Z. Yerong, Variational Born iteration method and its applications to hybrid inversion, IEEE Trans. Geosci. Remote Sensing, 38, 1709-1715, 2000. doi: 10.1109/36.851969.

[18] P. Berg and A. Abubakar, Contrast source inversion method: State of art, PIER, 189-218,2001. doi: 10.2528/PIER01061103.

[19] X. Ye and X. Chen, Subspace-based Distorted Born iterative method for solving Inverse scattering problems, IEEE Trans. Antennas Propagat., 72247232, 2017. doi: 10.1109/TAP.2017.2766658.

[20] N. Kinayman and M. I. Aksun, Modern Microwave Circuits, Artech House, 2005

[21] W. C. Chew., Waves and fields in inhomogenous media, IEEE Press Series on Electromagnetic Waves, 1990.

[22] R. E. Akdoğan., İki parçalı uzaya gömülü üç boyutlu cisimlere ilişkin ters saçılma probleminin Born yaklaşımı tabanlı ve kontrast kaynak tabanlı yöntemlerle çözümü. Yüksek Lisans Tezi, Niğde Ömer Halisdemir Üniversitesi Fen Bilimleri Enstitüsü, Türkiye, 2020.

[23] W.E. Patitz, B.C. Brock, E. G. Powell., "Measurement of Dielectric and Magnetic Properties of Soil", Sand1a report sand95-2419,UC-706 Unlimited Release 1995 